\title{
Emergency contraception with levonorgestrel: one hormone better than two
}

This is an exciting time for emergency contraception - a new method has arrived, we know more about how to optimise treatment, and new distribution channels are opening up. Combined with the political will, these advances should significantly reduce the burden of unwanted pregnancies.

One of the important new ingredients is the WHO trial demonstrating the superior efficacy of levonorgestrel used on its own; the pregnancy rate with levonorgestrel alone was a third that of the comparison group using the traditional Yuzpe regime. ${ }^{1}$ The study was the largest of its kind, with almost 2000 participants, had a secure method of randomisation, and was blinded to all participants. Two study groups were thus created which differed only in the type of emergency contraception used, thereby minimising the possibilities for random error and bias. The result, however, has been questioned on a number of accounts. The Yuzpe regime did not appear to perform particularly well in the trial and this might have influenced the comparison. ${ }^{2,3}$ But the method used to estimate the 'true efficacy' of emergency contraception, or proportion of pregnancies prevented, has limitations, although, as it was applied equally to both arms of the trial, it should not have biased the result.

In the absence of placebo controlled trials, external controls, derived from published studies in which no contraception was used, are used to provide an estimate of the number of 'expected pregnancies', based on fecundity on each day of the menstrual cycle. ${ }^{4-6}$ However, these calculations assume that the day of ovulation can be accurately determined from retrospective reporting of day of the last menstrual period and cycle length. A recent study found a poor correlation between menstrual data and hormonal status on the day of emergency treatment. ${ }^{7}$ Not surprisingly, studies of emergency contraception frequently find pregnancies occurring when none are expected, using what is, in effect, the rhythm method to estimate risk of pregnancy. In the WHO study the external controls used were older and some desired pregnancy, while almost half of the women in the trial had accidents with a barrier contraceptive, often with a spermicide. Other differences from the external controls are likely. Applying these externally derived estimates to randomised trials opens too many doors through which error can enter into otherwise well-conducted studies.

Because of its unreliability, this attempt to correct for a possible imbalance in risk of pregnancy should be dropped from randomised trials which will distribute women of equal risk of pregnancy to each arm, if the numbers are large enough, as indeed they were in the recent WHO trial. We cannot tell the so-called 'true effectiveness' of each method, but we can tell reliably if one is better than the other, and by how much - precisely the sort of information required for clinical decision making.

The opportunity to determine the true efficacy of Yuzpe was lost as the early workers, as is common, felt it unethical to conduct a placebo controlled trial. ${ }^{8}$ Had one been organised by the doubters, the value of the Yuzpe regime should have been apparent and the uptake of emergency contraception greater. Archie Cochrane lamented the way in which medical interventions become established without a randomised trial being conducted, so that it is then regarded as unethical for a trial to be set up. The only way to avoid this, in his opinion, is to randomise the first patient whenever a new treatment is introduced. ${ }^{9}$ In family planning we have many practices which have not been assessed, having taken what Muir Gray calls, 'the evaluation bypass'.

Caution in accepting the WHO trial results has also been advised because it has been said that fewer women in the Yuzpe arm met the study criteria for 'correct use'. 3 This is not supported in the data. Similar numbers in both groups used the method correctly, and amongst these women the Yuzpe pregnancy rate was halved in the levonorgestrel arm. The pregnancy rate following both treatments was higher following incorrect use, but again the progestogen method was superior; indeed, the benefits of levonorgestrel were even greater following incorrect use. It appears that the traditional Yuzpe method is less tolerant to variations in the regimen, a common experience in everyday practice. Unknown to the investigators, a few women were already pregnant at the time of treatment, and more of these were in the Yuzpe group. However, removing these women from the analysis does not effect the comparative advantage of levonorgestrel.

It is interesting that the part of the WHO study that has been given most credence $\mathrm{e}^{3,10}$ is the result with the lowest validity - the shorter the coitus to treatment interval, the lower the pregnancy rate. ${ }^{1}$ It is based on non-experimental observations, albeit from a randomised trial. Clinical practice has been heavily influenced by an earlier pooling of observational studies of the Yuzpe regimen, of variable quality, which found a similar pregnancy rate regardless of the day of treatment. ${ }^{11}$ Adding the WHO data to the earlier studies of the Yuzpe method gives a combined pregnancy rate for Days one to three of 1.7, 2.8 and 2.6, respectively. The result is statistically significant $(\mathrm{p}=0.05)$, but not when adjusted for the effects of study site $(\mathrm{p}=0.1)$. There is a high degree of heterogeneity across studies, and the loss to follow-up rates are as high as $24 \%$, so it is questionable if the pooling exercise is justified. Given the small number of pregnancies reported in the studies, any unrecorded pregnancies could have a considerable impact on the results. The rigorous methods of the WHO trial, and the low loss to follow-up rate $(1.4 \%)$, probably avoided some of the biases in the earlier studies, but in the absence of randomisation to different periods of delay it remains possible that the more fertile women presented later for treatment.

So how do we advise women? Our previous advice that delay in treatment was a secondary concern was based on more questionable data. The close relationship in the WHO study between interval to treatment and pregnancy rate is impressive. It appears that intuition - the sooner the treatment the better - is probably correct. We were right to say that it is not just for the 'morning after', ${ }^{12}$ but it may 
still be the best time. Better still to have a packet in your pocket or in the medicine cupboard, and studies in advanced prescribing are particularly interesting in this regard. The pregnancy rate in the WHO trial in almost 400 women who started either treatment within 12 hours of intercourse was $0.5 \%$ (95\% confidence intervals, $0 \%$ to $1.2 \%) .^{13}$ The combined data from the WHO and Ho and Kwan ${ }^{14}$ trials suggest that the effect of delay may be different for levonorgestrel and the Yuzpe method, and that early treatment is even more important with the latter.

The data to date strongly support the superior efficacy of the progestogen-only method. A Cochrane meta-analysis of the two trials of levonorgestrel versus the Yuzpe regime found a halving of the pregnancy rate with levonorgestrel (odds ratios $0.51,95 \%$ confidence intervals 0.31 to 0.84 ). ${ }^{15}$ The Ho and Kwan study excluded women seeking treatment more than 48 hours after intercourse. ${ }^{14}$ Combining data for first and second day treatments only gives a similar result. Both trails have also demonstrated that levonorgestrel is better tolerated. However, we finally have evidence of an effective prophylactic treatment for nausea and vomiting induced by the Yuzpe regime. ${ }^{16}$ The only other randomised study, a trial of eating before the first dose of Yuzpe, suggested no benefit, although the study was small and had a high loss to follow-up rate. ${ }^{17}$

What will the impact of levonorgestrel be on unwanted pregnancies? Women with irregular cycles or using hormones were excluded in the two trials comparing levonorgestrel to the Yuzpe method. There were around 800000 prescriptions for Schering PC4 in England in 1998. Let us assume that $50 \%$ of these $(400000)$ were to women with regular cycles and who were not using hormonal contraception. If we also assume a pregnancy rate of about $2 \%$ following Yuzpe use, around 8000 unplanned pregnancies will have occurred in regular cycling women that year. Had levonorgestrel been used we would expect 4000 fewer pregnancies. Levonorgestrel may well be superior to Yuzpe for the other 50\% with lower risk also, but it is not possible to say.

There are three obstacles to the wholesale switch to levonorgestrel: cost, difficulty in distribution relating to its new drug status, and resistance to change. The new product, Levonelle-2, has been introduced at an unnecessary high price. Knowledge of its value has come from mostly publicly funded research, and the product will sell itself. A similar product is available in many countries at a fraction of the UK price.

Contraceptives are increasingly supplied by nurses working to group protocols. As with all new drugs, Levonelle-2 carries a black triangle and doctors and hospital pharmacists are asked to report all suspected reactions. Newly introduced drugs are normally excluded from group protocols. ${ }^{18}$ There are exceptions however, and levonorgestrel may qualify if proper structures are set up for reporting adverse effects, and if its use is consistent with best practice - another reason to be clear about the superior efficacy of levonorgestrel.

The evidence favours levonorgestrel over the Yuzpe regime. Yet prescribing behaviour frequently lags behind best evidence. ${ }^{19}$ The UK has been a leader in the provision of emergency hormonal contraception, with exponential growth in prescriptions since the mid 1980's. The speed with which levonorgestrel for emergency use has been introduced in this country following a high quality randomised trial is impressive. The challenge now is to implement the research findings and make a better emergency method a reality for women. The use of levonorgestrel emergency contraception may be an appropriate service standard for family planning in the future.

Statements on funding and competing interests Funding: None.

Competing interests: None.

Paul A O'Brien, MB

Senior Clinical Medical Officer in Clinical Effectiveness, Services for Women, Parkside Health NHS Trust, St Charles Hospital, Exmoor St, London, W10 6DZ

PaulOBrien@care.prestel.co.uk

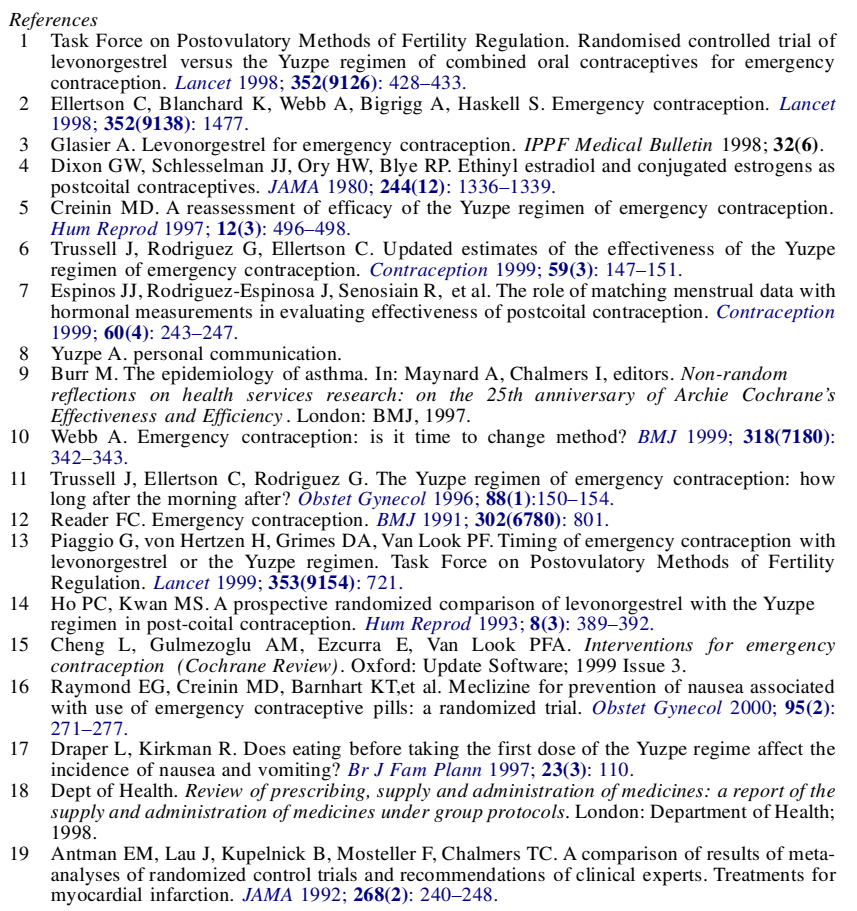

Task Force on Postovulatory Methods of Fertility Regulation. Randomised controlled trial of evonorgestrel versus the Yuzpe regimen of combined oral contraceptives for emergenc (E) JJ, Ory HW, Blye RP. Ethiny postcoital contraceptives. JAMA 1980; 244(12): 1336-1339.

Hum Reprod 1997; 12(3): 496-498.

Trussell J, Rodriguez G, Ellertson C. Updated estimates of the effectiventes

Espinos JJ, Rodriguez-Espinosa J, Senosiain R, et al. The role of matching menstrual data with 999; 60(4): 243-247.

Burr M. The epidemiology of asthma. In: Maynard A, Chalmers I, editors. Non-random

Tussell J, Ellertson C, Rodriguez G. The Yuzpe regimen of emergency contraception: how ong after the morning after? Obstet Gynecol 1996; 88(1):150-154.

Piaggio G, von Hertzen $\mathrm{H}$, Grimes DA, Van Look PF. Timing of emergency contraception with regimen in post-coital con

Cheng L, Gulmezoglu AM, Ezcurra E, Van Look PFA. Intententio

waynond EG, Creinin MD, Barnhan KT,et al. Mechizine for prevention of na

Draper L, Kirkman R. Does eating before taking the first dose of the Yuzpe regime affect the Dept of Hellh. Review of prescibing,

Antman EM, Lau J, Kupelnick B, Mosteller F, Chalmers TC. A comparison of results of metanalyses of randomized control trials and recommendations of clinical experts. Treatments fo
myocardial infarction. JAMA 1992; 268(2): 240-248.

\section{Volume numbers to start in January}

From 2000 onwards each volume of The British Journal of Family Planning (comprising four issues) will start in the January of each year. This issue is thus Volume 26, Number 2, April 2000. This replaces the older system whereby the Volumes started in the April of each year, and readers should note that this change means that there is no Volume 25, Number 4 of this Journal. 